\title{
The oncometabolite 2-hydroxyglutarate produced by mutant IDH1 sensitizes cells to ferroptosis
}

\author{
Tian-Xiang Wang ${ }^{1}$, Jun-Yun Liang ${ }^{1}$, Cheng Zhang ${ }^{1}$, Yue Xiong ${ }^{2}$, Kun-Liang Guan $\mathbb{1}^{3}$ and Hai-Xin Yuan (1)
}

\begin{abstract}
Ferroptosis is a non-apoptotic form of cell death characterized by the iron-dependent lipid peroxidation and is implicated in several human pathologies, such as tissue ischemia, neurodegeneration, and cancer. Ferroptosis appears to be high cell-context dependent and the regulation of ferroptosis by physiological or pathological conditions are unclear. Here, we report that tumor-derived IDHI mutation sensitizes cells to ferroptosis. Deletion of the mutant IDHI allele in IDH1 heterozygous tumor cells or pharmacological inhibition of mutant IDH1 to produce the oncometabolite D-2-hydroxyglutarate (D-2-HG) confers resistance to erastin-induced ferroptosis. Conversely, ectopic expression of mutant IDH1 or treatment of cells with cell-permeable D-2-HG promotes the accumulation of lipid reactive oxygen species (ROS) and subsequently ferroptosis. Mechanistically, mutant IDH1 reduces the protein level of the glutathione peroxidase 4 (GPX4), a key enzyme in removing lipid ROS and ferroptosis, and promotes depletion of glutathione. Our results uncover a new role of mutant IDH1 and 2-HG in ferroptosis.
\end{abstract}

\section{Introduction}

Ferroptosis is a recently identified non-apoptotic form of regulated cell death that is prone to happen in tumor cells bearing Ras gene mutation ${ }^{1}$ or highly transformed tumor cells ${ }^{2}$. Ferroptosis is distinct from apoptosis or necroptosis based on the fact that caspase or RIPK1 inhibitors do not hinder ferroptosis process. Ferroptosis also displays unique morphological features such as shrunken mitochondria and increased mitochondrial membrane density ${ }^{3}$. Although the physiological functions of ferroptosis remains elusive, much efforts have been taken in recent years to elucidate the mechanisms underlying ferroptosis. It is believed that excessive accumulation of lipid peroxide (lipid ROS), generated by the family of lipoxygenases, is a critical cause leading to

\footnotetext{
Correspondence: Hai-Xin Yuan (yuanhaixin@fudan.edu.cn)

${ }^{1}$ The Fifth People's Hospital of Shanghai and the Molecular and Cell Biology Research Lab of the Institutes of Biomedical Sciences, Fudan University, Shanghai, China

${ }^{2}$ Department of Biochemistry and Biophysics, Lineberger Comprehensive Cancer Center, University of North Carolina at Chapel Hill, Chapel Hill, North Carolina, USA

Full list of author information is available at the end of the article.

Edited by G. Raschellà
}

ferroptosis ${ }^{4}$. This links ferroptosis with the breakdown of cellular redox homeostasis maintained by glutathione and glutathione peroxidase 4 (GPX4), the only enzyme in mammalian cells that could eliminate lipid ROS using reduced glutathione (GSH) as a substrate. Accordingly, compounds that inhibit the lipoxygenases such as Nordihydroguaiaretic acid (NDGA) and zileuton are effective in suppressing ferroptosis ${ }^{5}$. On the other hand, compounds that inhibit cystine-glutamate antiporter (system $\mathrm{Xc}^{-}$) and subsequently lower GSH level (such as erastin) or that inhibit GPX4 activity (such as RSL3) strongly induce ferroptosis.

Besides system $\mathrm{Xc}^{-}$(SLC7A11) and GPX4, several other genes have also been reported to influence cells' sensitivity to ferroptosis including ACSL4, TP53 and GLS2 ${ }^{6-8}$. These studies linked ferroptosis to multiple cellular processes like iron homeostasis, redox homeostasis, lipid metabolism, and glutaminolysis. However, the fact that ferroptosis is highly dependent on cell-type indicates the complex mechanisms underlying ferroptosis. Given highly transformed and drug-resistant tumor cells are prone to ferroptosis, it is of great importance to understand the mechanism underlying ferroptosis and apply it to

\section{(c) The Author(s) 2019}

(c) (i) Open Access This article is licensed under a Creative Commons Attribution 4.0 International License, which permits use, sharing, adaptation, distribution and reproduction c. in any medium or format, as long as you give appropriate credit to the original author(s) and the source, provide a link to the Creative Commons license, and indicate if changes were made. The images or other third party material in this article are included in the article's Creative Commons license, unless indicated otherwise in a credit line to the material. If material is not included in the article's Creative Commons license and your intended use is not permitted by statutory regulation or exceeds the permitted use, you will need to obtain permission directly from the copyright holder. To view a copy of this license, visit http://creativecommons.org/licenses/by/4.0/. 
personalized anti-cancer strategies and some other diseases such as $I / R$ injury and even neuron degenerative diseases $^{9-11}$.

Hot-spot mutations of the metabolic enzyme IDH1/2 (isocitrate dehydrogenases 1 and 2) are observed in several types of cancers including secondary glioblastomas $(\mathrm{GBMs})^{12}$, acute myeloid leukemia $(\mathrm{AML})^{13}$, chondrosarcoma $^{14-16}$ and cholangiocarcinoma ${ }^{17,18}$. The high frequent mutations on the single residue (R132 in IDH1, R172 or R140 in IDH2) have caught broad interest in its function in promoting tumor development. Diseaseassociated IDH1/2 mutations not only abolish their enzyme activity in converting isocitrate to $\alpha-K G$, but also endow the mutant protein with a new activity to convert $\alpha$-KG to (D)-2-hydroxyglutarate (2-HG) using NADPH as co-factor [reviewed in ref. ${ }^{19}$ ]. In tumor cells bearing IDH mutations, 2-HG can be accumulated at concentrations of millimolar level $^{20}$, at which it competitively inhibits multiple $\alpha-\mathrm{KG} / \mathrm{Fe}(\mathrm{II})$-dependent dioxygenases ${ }^{21,22}$, consequently interfering many relevant processes as epigenetic regulation, genetic instability, $\mathrm{T}$ cell differentiation and tumor immunity [reviewed in ref. ${ }^{19}$ ]. Interestingly, 2HG was also found to suppress cell growth through binding and inhibiting ATP synthase, a function that $\alpha$ KG also executes, suggesting complex relationship between the two metabolites ${ }^{23,24}$. In addition, elevated 2HG has also been observed in tumor cells lacking an IDH mutation and even in non-tumorigenic heart during ischemic preconditioning and hematopoietic stem cells after disruption of the respiratory chain [reviewed in ref. ${ }^{19}$ ]. These studies demonstrate $2-\mathrm{HG}$ is involved in pathological conditions not limited to cancer.

Here, we examined the relationship between mutant IDH1 and ferroptosis sensitivity in response to erastin. We find that high level of 2-HG resulting from $I D H 1^{R 132 C}$ mutation sensitizes cells to erastin-induced ferroptosis. In detail, $I D H 1^{R 132 C}$ mutation and its metabolic product 2HG could decrease the protein level of GPX4 and result in a rapid exhaustion of glutathione upon erastin. Our results present a novel role of tumor-derived IDH1 mutation and oncometabolite 2-HG in ferroptosis.

\section{Materials and methods}

\section{Antibodies, plasmid, and chemicals}

Antibodies against Flag (ShanghaiGenomics), $\beta$-actin (Genescript), GPX4 (Abcam), ACSL4 (Proteintech), ERK (CST), p-ERK (CST), NRF2 (Abcam) were purchased commercially.

Full-length cDNA of $D 2 H G D H$ and $I D H 1$ was amplified by PCR and cloned into indicated pBabe and pQCXIH. Point mutations for $I D H 1$ were generated by site-directed mutagenesis and verified by Sanger sequencing.

AG-120 (CSNpharm), IDH-889 (DC Chemicals), erastin (MedChemExpress, MCE), RSL3 (MCE), Deferoxamine mesylate (MCE), Ferrostatin-1 (Selleck Chemicals), (2 R)2-Hydroxyglutaric Acid Octyl Ester Sodium Salt, and (2S)-2-Hydroxyglutaric Acid Octyl Ester Sodium Salt (Toronto Research Chemicals) were purchased commercially.

\section{Cell culture, transfection, and stable cell lines generation}

HEK293T, HT-1080 and KYSE-170 cells were purchased from the American Type Culture Collection (ATCC). HEK293T and HT-1080 cells were cultured in DMEM (Invitrogen) supplemented with 5\% FBS (Gibco), $100 \mathrm{unit} / \mathrm{mL}$ penicillin, and $100 \mathrm{mg} / \mathrm{mL}$ streptomycin (Gibco). KYSE-170 cells were cultured in RPMI 1640 medium (Gibco) with $10 \%$ FBS, $100 \mathrm{unit} / \mathrm{mL}$ penicillin, and $100 \mathrm{mg} / \mathrm{mL}$ streptomycin.

Cell transfection was carried out by Lipofectamine 2000 according to the manufacturer's protocol (Invitrogen).

Cells stably expressing the indicated proteins were established by standard retroviral infection, and selected in $2 \mathrm{mg} / \mathrm{mL}$ puromycin (Ameresco) or $50 \mathrm{mg} / \mathrm{mL}$ hygromycin B (Ameresco) for 7 days. The mutant IDH1 allele knocked out HT-1080 $\left(\mathrm{IDH} 1^{+/-}\right)$cells were generated previously by TALEN technology.

\section{Cell viability assay}

Cells were seeded into 12-well plates and incubated with the indicated treatments. Subsequently, cells were digested with trypsin and collected for trypan blue staining. Cell viability was counted and calculated by using an automated cell counter (Count Star, IC 1000). Cell viability under test conditions is reported as a percentage relative to the negative control treatment.

\section{Measurement of intracellular glutathione level}

Cells were seeded into 6-well dishes at a concentration of 400,000 cells/well. After one day, cells were treated with erastin for the indicated times and harvested by trypsinization. Cell numbers were counted by using an automated cell counter (Count Star, IC 1000). Intracellular glutathione level was detected using the Total Glutathione Assay Kit (Beyotime, S0052) following the manufacture's instructions.

\section{Cellular lipid ROS assay}

Cells were seeded into 6-well dishes at a concentration of 400,000 cells/well. After one day, cells were treated with erastin for the indicated times. After treatment, cells were stained with C11-BODIPY 581/591 (Thermo Fisher Scientific, D3861) for $30 \mathrm{~min}$ at $37^{\circ} \mathrm{C}$ and then harvested by trypsinization. Cells were re-suspended in PBS and strained through a 40- $\mu \mathrm{m}$ cell strainer (BD Falcon), and then analyzed cells using flow cytometer (Accuri C6, BD Biosciences) equipped with $488 \mathrm{~nm}$ laser for excitation. Data were collected from the FL1. A minimum of 10,000 cells were analyzed per condition. 


\section{Metabolite extraction and GC-MS analysis}

Confluent cells in 6-well plated were homogenized in $0.5 \mathrm{~mL}$ of chilled $80 \%(\mathrm{v} / \mathrm{v})$ methanol. The samples were centrifuged at 12,000 rpm for $10 \mathrm{~min}$ and the supernatants were transferred to a high recovery glass sampling vial (CNW, VAAP-31509-1232-100) to vacuum dry at room temperature. The residue was re-suspended with $30 \mu \mathrm{L}$ pyridine containing $20 \mathrm{mg} / \mathrm{mL}$ methoxyamine hydrochloride (Sigma-Aldrich, 226904) at $37^{\circ} \mathrm{C}$ overnight and further derivatized with $20 \mu \mathrm{L}$ of $\mathrm{N}$-tert-Butyldimethylsilyl-N-methyltrifluoroacetamide (Sigma-Aldrich, Cat\#: 394882 ) at $70^{\circ} \mathrm{C}$ for $30 \mathrm{~min}$. Then $1 \mu \mathrm{L}$ aliquot of the derivatized sample was injected into Agilent 7890 A gas chromatography coupled with Agilent 5975 C mass spectrometer. Separation was achieved on a HP- $5 \mathrm{~ms}$ fused-silica capillary column with the helium as the carrier gas at a constant flow rate of $1 \mathrm{~mL} / \mathrm{min}$ through the column.

\section{Statistical analysis}

Statistical analyses were performed using GraphPad Prism 6.0c. All data shown represent the results obtained from triplicated independent experiments with standard deviation of the mean (mean $\pm \mathrm{SD}$ ). The sample sizes were chosen to allow for statistical significance testing assuming a major effect and a small variation. The variance was similar between the compared groups. The $p$ values were calculated with two-tailed unpaired Student's $t$-test or one-way ANOVA with Dunnett's multiple comparisons test or Bonferroni's multiple comparisons test as indicated in corresponding figure legends. $p<0.05$ were considered statistically significant.

\section{Results}

\section{Mutant IDH1 promotes erastin-induced ferroptosis}

HT-1080 is a model cell line extensively used for ferroptosis study because of its high sensitivity to ferroptosis-inducing compounds, such as erastin and RSL $^{25}$. HT-1080 cells harbor Ras mutation that makes cells prone to ferroptosis. Notably, HT-1080 also bears a heterozygous $\mathrm{R} 132 \mathrm{C}$ mutation in IDH1 [referred to as HT1080 $\left.\left(I D H 1^{+/ R 132 C}\right)^{26}\right]$. No report has linked IDH1 mutation with ferroptosis by now. We previously generated HT-1080 cell lines with the mutant IDH1 allele knocked out [referred to as HT1080 $\left(\mathrm{IDH1}^{+-}\right)$] and then putting back wild-type, R132C single mutant or R132C/ T77A double mutant of IDH1 gene. Among them, R132C/T77A double mutant inactivates the activity of mutant IDH1 to produce D-2-HG ${ }^{27}$. We performed ferroptosis assays in these isogenic cell lines following treatment with erastin. Surprisingly, deletion of $I D H 1^{\mathrm{R} 132 \mathrm{C}}$ mutant allele significantly reduced erastininduced ferroptosis (Fig. 1a). Compared to the parental HT1080 $\left(I D H 1^{+/ R 132 C}\right)$ cells, cell viability was increased by
2.5 folds in HT1080 $\left(I D H 1^{+/-}\right)$cells (Fig. 1b). Reexpression of the $\mathrm{R} 132 \mathrm{C}$ mutant of $I D H 1$, accumulated D-2-HG (Fig. 1c) and restored cells' sensitivity to erastin (Fig. 1b), indicating the production of 2-HG is required for HT-1080 cells' sensitivity to erastin. Though significantly rescuing cell death compared with $\mathrm{R} 132 \mathrm{C}$ mutant, re-expression of R132C/T77A double mutant still partially restored cells' sensitivity to erastin (Fig. 1b). This data indicated besides 2-HG, IDH1 mutant may have additional mechanism to promote ferroptosis. We confirmed that erastin-induced cell death was ferroptosis using an iron chelator, deferoxamine (DFO), and a ferroptosis inhibitor, Ferrostatin-1 (Fig. 1d, e).

To further confirm the role of $I D H 1^{\mathrm{R} 132 \mathrm{C}}$ mutation in enhancing erastin-induced ferroptosis, we overexpressed wild-type or R132C mutant IDH1 in KYSE-170 esophagus tumor cells which contain two wild-type $I D H 1$ alleles (Fig. 1f). Consistently, overexpression of IDH $1^{\mathrm{R} 132 \mathrm{C}}$ promoted erastin-induced ferroptosis while wide type IDH1 overexpression exerted no effect on cells' sensitivity to erastin (Fig. 1g). We also treated HT-1080 cells with two small molecules that specifically inhibit mutant IDH1, AG-120 (Ivosidenib) $^{28}$ and IDH- $889^{29}$, and found that both inhibitors reduced cells' sensitivity to erastin (Fig. 1h). Together, these data demonstrate that IDH1 $1^{\mathrm{R} 132 \mathrm{C}}$ mutation promotes cells' sensitivity to erastin-induced ferroptosis.

\section{Mutant IDH1 enhances erastin-induced lipid ROS accumulation}

Excessive accumulation of lipid ROS is a critical cause of ferroptosis which could be detected by using fluorescent radio-probe C11 BODIPY 581/591. To determine whether mutant IDH1 could promote cells' sensitivity to erastin by increasing lipid ROS, we measured the lipid ROS levels in HT-1080 cells with different genotypes of IDH1. As shown in Fig. 2a, lipid ROS began to accumulate at $8 \mathrm{~h}$ in HT-1080 $\left(I D H 1^{+/-}\right)$cells ectopically expressing $I D H 1^{\mathrm{R} 132 \mathrm{C}}$ and rapidly increased at $10 \mathrm{~h}$ after erastin addition. At $10 \mathrm{~h}$, approximately $80 \%$ of the cells showed high level of lipid ROS. In contrast, there was no significant increase of lipid ROS in HT-1080 $\left(I D H 1^{+/-}\right)$cells ectopically expressing wide-type $I D H 1$ in the same duration.

We next determined the effect of IDH1 mutation to different doses of erastin. We found that $5 \mu \mathrm{M}$ of erastin strongly induced lipid ROS accumulation in cells expressing IDH1 mutant, but not in cells expressing wildtype $I D H 1$. At a lower dose of erastin $(1 \mu \mathrm{M})$, there was no significant accumulation of lipid ROS (Fig. 2b). Consistent with this, $1 \mu \mathrm{M}$ of erastin did not induce cell death while $5 \mu \mathrm{M}$ of erastin was able to cause ferroptosis in cells expressing $\mathrm{R} 132 \mathrm{C}$ mutant, but not wild-type IDH1 (Fig. 2c). In addition, IDH1 mutant inhibitors AG-120 and 
A

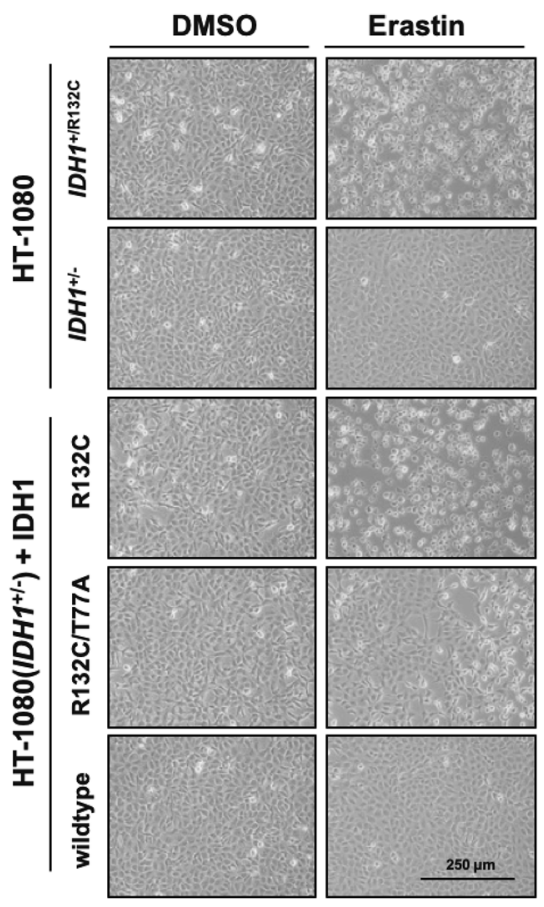

D

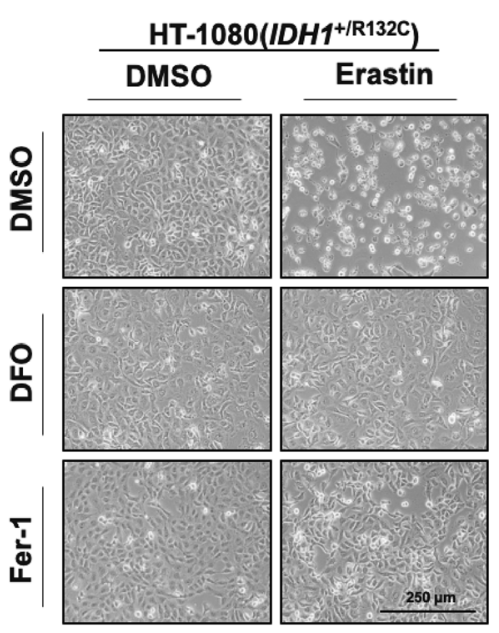

G

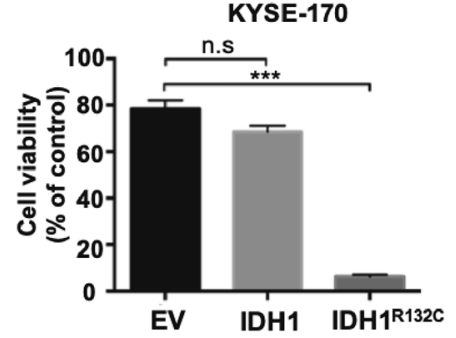

B

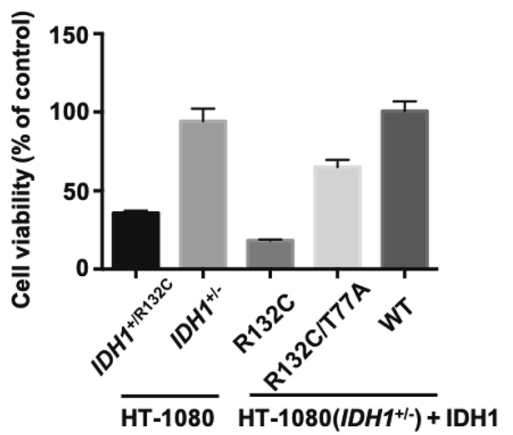

C

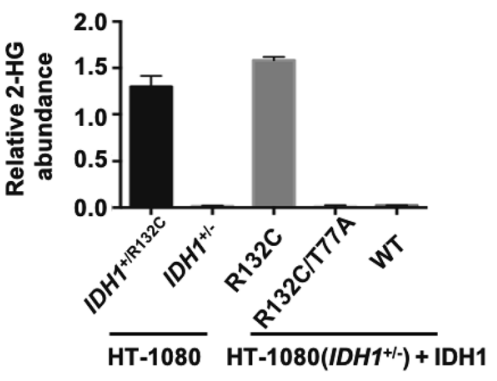

E
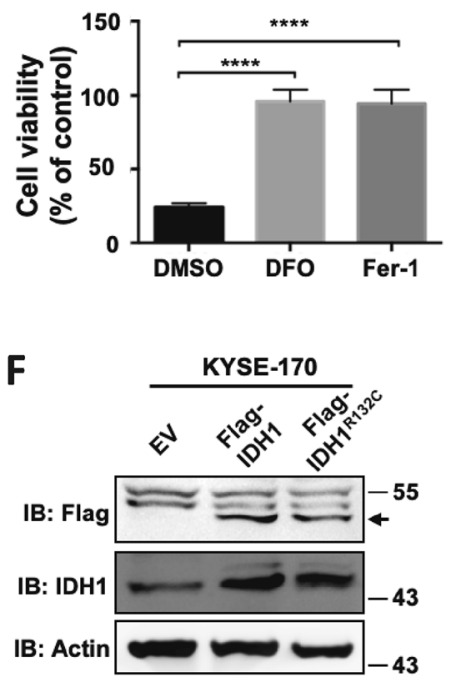

H

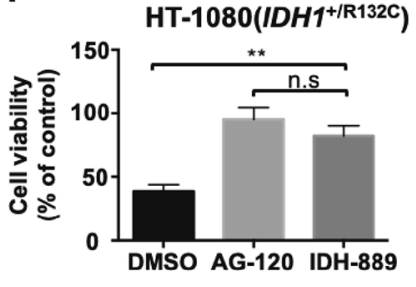


(see figure on previous page)

Fig. 1 Mutant IDH1 promotes erastin-induced ferroptosis. a IDH1 $1^{\mathrm{R} 132 \mathrm{C}}$ mutation promotes erastin-induced ferroptosis. HT-1080 cells with indicated IDH1 genotypes were treated with erastin $(10 \mu \mathrm{M})$ for $12 \mathrm{~h}$, and microscopically photographed. b Cell viability in (a) were assayed by trypan blue staining. $\mathbf{c}$ Endogenous 2-HG levels of HT-1080 cells with different IDH1 genotypes in (a) were determined by GC-MS. d Ferroptosis induction in HT-1080 cells. HT-1080 cells were treated with erastin, DFO, and Fer-1 as indicated for $12 \mathrm{~h}$, and microscopically photographed. e Cell viability in (d) were assayed by trypan blue staining $\left(n=3 ;{ }^{* * * *} p<0.0001\right.$ vs. DMSO group, Dunnett's multiple comparisons test). $\mathbf{f}$ Expression of Flag-IDH1 or Flag- $I D H 1^{\mathrm{R} 132 \mathrm{C}}$ in KYSE-170 cells were detected by Western blot. $\mathbf{g} \mathrm{IDH} 1^{\mathrm{R} 132 \mathrm{C}}$ mutation promotes erastin-induced ferroptosis in KYSE-170 cells. KYSE170 cells expressing Flag-IDH1 or Flag-IDH1 ${ }^{\mathrm{R} 132 \mathrm{C}}$ were treated with erastin for $16 \mathrm{~h}$ and cell viability was assayed by trypan blue staining $\left(n=3 ;{ }^{* * *} p<\right.$ 0.001 vs. EV group, Dunnett's multiple comparisons test). $\mathbf{h}$ Pharmacological inhibition of mutant IDH1 reduces cells' sensitivity to erastin. HT-1080 cells were treated with AG-120 or IDH-889 for $12 \mathrm{~h}$, and then with erastin for additional $12 \mathrm{~h}$. Cell viability were assayed by trypan blue staining $(n=3$; ${ }^{* *} p<0.01$ and ${ }^{* * *} p<0.001$, Bonferroni's multiple comparisons test)

IDH-889 also suppressed erastin-induced lipid ROS accumulation in $\mathrm{HT} 1080\left(\mathrm{IDH} 1^{+/ \mathrm{R} 132 \mathrm{C}}\right)$ cells (Fig. 2d). Collectively, these data indicate that mutant IDH1 promotes erastin-induced ferroptosis through increasing lipid ROS accumulation in a catalytic-dependent manner.

\section{D-2-HG promotes erastin-induced ferroptosis}

$\mathrm{IDH} 1^{\mathrm{R} 132 \mathrm{C}}$ mutant confers a neomorphic enzymatic gainof-function to convert $\alpha-K G$ to D-2-HG. High level of $2-\mathrm{HG}$ was detected in cells expressing mutant $I D H 1^{\mathrm{R} 132 \mathrm{C}}$ (Fig. 1c). Ectopic expression of R132C mutant in HT-1080 $\left(I D H 1^{+/-}\right)$cells, but not the R132C/T77A double mutant which inactivates the production of 2-HG re-sensitized cells to erastin-induced ferroptosis. To directly demonstrate the role of 2-HG in enhancing ferroptosis, we overexpressed D2-HG dehydrogenase (D2HGDH), which catalyzes the oxidation of D-2-HG to $\alpha-K G$ and thus reduces D-2-HG level in HT-1080(IDH1 ${ }^{+/ R 132 C}$ ) cells (Fig. 3a). In line with this, ferroptosis induced by erastin was inhibited by D2HGDH overexpression (Fig. 3b, c). Next, we pretreated KYSE-170 cells with cell-permeable octyl-D- or L-2-HG followed by addition of erastin. Both enantiomers of 2-HG promoted erastin-induced ferroptosis in KYSE-170 cells (Fig. 3d, e). Although D-2-HG showed higher potency in promoting ferroptosis than L-2-HG when used at the same concentration, we found that L-2-HG accumulation within cells was only about $10 \%$ of D-2-HG after $24 \mathrm{~h}$ treatment (Fig. 3f). In accordance, cell-permeable octyl-D-2-HG promoted HT-1080 $\left(I D H 1^{+/-}\right)$cells' sensitivity to erastin (Fig. 3g, h). Consistent with their role in promoting ferroptosis, D-2-HG caused a stronger lipid ROS accumulation (Fig. 3i). Based on these data, we conclude that D-2-HG serves as the effector of mutant IDH1 within cells to enhance cells' sensitivity to ferroptosis.

\section{Mutant IDH1 exacerbates erastin-induced glutathione depletion}

Erastin-induced ferroptosis through inhibiting cystine uptake and thus decreasing cellular concentration of cysteine which is the substrate for glutathione synthesis ${ }^{30}$. Moreover, reduced glutathione (GSH) is indispensable for the enzymatic activity of $\mathrm{GPX} 4^{31}$. To test whether
IDH $1^{\mathrm{R} 132 \mathrm{C}}$ mutant influences erastin-induced exhaustion of glutathione, we determined the cellular levels of cysteine, glycine, and L-glutamic acid using GC-MS. After treatment with erastin for $5 \mathrm{~h}$, cellular cysteine level decreased to about ten percent of that at steady state, and then it maintained at this level up to $10 \mathrm{~h}$ after erastin treatment (Fig. 4a). Glycine and glutamic acid, the other two amino acids used for glutathione synthesis, remained stable in response to erastin treatment. The reduction of cysteine showed no difference between HT$1080\left(\mathrm{IDH} 1^{+/ \mathrm{R} 132 \mathrm{C}}\right)$ and $\mathrm{HT}-1080\left(\mathrm{IDH} 1^{+/-}\right)$cells after erastin treatment, indicating the cysteine reduction is unlikely to be the direct cause for the different sensitivity to erastin between these two cell lines.

Next, we measured the endogenous glutathione level. During the early phase $(0-4 \mathrm{~h})$ after erastin addition, cellular glutathione level dropped by about $50 \%$. Then the endogenous glutathione level continued to drop during late phase $(6-10 \mathrm{~h})$ after erastin addition (Fig. 4b). We found the basal level of glutathione in HT-1080 $\left(\mathrm{IDH1}^{+/-}\right)$ cells was lower than that in HT-1080(IDH1 $\left.1^{+/ \mathrm{R} 312 \mathrm{C}}\right)$ cells. Erastin treatment caused a decline of glutathione level in both HT-1080 $\left(\mathrm{IDH} 1^{+/ \mathrm{R} 132 \mathrm{C}}\right)$ and $\mathrm{HT}-1080\left(\mathrm{IDH} 1^{+/-}\right)$ cells, but notably the rate of decline was much lower in HT-1080 $\left(I D H 1^{+/-}\right)$cells (Fig. 4c). Glutathione level was not detectable after $12 \mathrm{~h}$ in HT-1080(IDH1 $\left.{ }^{+/ \mathrm{R} 132 \mathrm{C}}\right)$ cells as most cells died then (Fig. 4d). Erastin-induced ferroptosis is thought as a process resulted from gradually decreasing glutathione which leads to the accumulation of lipid ROS. Our results suggest the expression of mutant IDH1 may accelerate the glutathione depletion and lipid ROS accumulation, and thereby promote ferroptosis.

\section{IDH1 mutation reduces GPX4 protein level}

The above data establish a role of IDH $1^{\mathrm{R} 132 \mathrm{C}}$ mutant and its product D-2-HG in enhancing ferroptosis. However, time-course experiments demonstrated that suppression of $\mathrm{IDH} 1^{\mathrm{R} 132 \mathrm{C}}$ by deletion of mutant allele or inhibitor treatment slowed but did not abolish ferroptosis (Fig. 5a). Interestingly, we also observed that if erastin was removed after $12 \mathrm{~h}$ treatment, HT-1080 $\left(I D H 1^{+/ R 132 C}\right)$ cells continue to undergo ferroptosis while HT-1080 


\section{A}
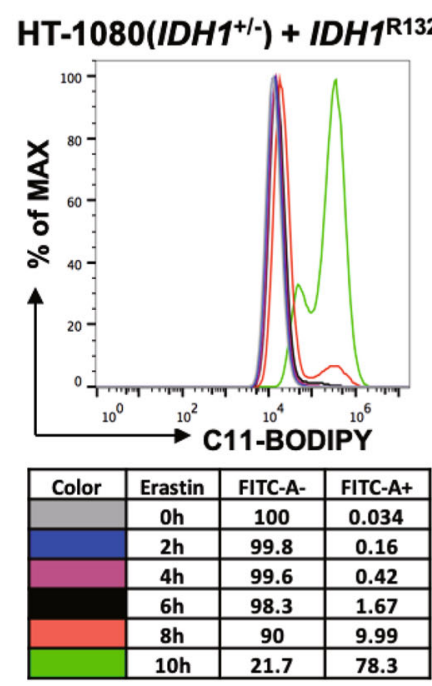

B

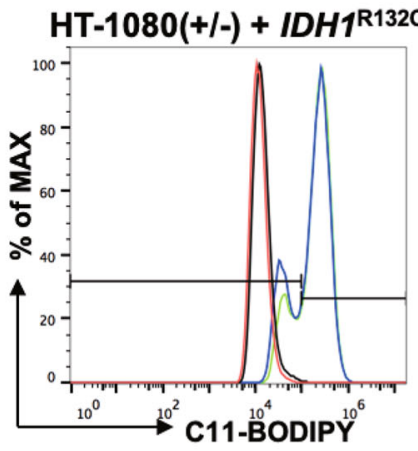

\begin{tabular}{|c|c|c|c|}
\hline Color & Erastin & FITC-A- & FITC-A+ \\
\hline & - & 99.9 & 0.08 \\
\hline & $1 \mu \mathrm{M}$ & 99.7 & 0.33 \\
\hline & $5 \mu \mathrm{M}$ & 29.1 & 70.9 \\
\hline & $10 \mu \mathrm{M}$ & 22.8 & 77.2 \\
\hline
\end{tabular}

$\mathrm{HT}-1080\left(\mathrm{IDH}^{++\cdot-}\right)+\mathrm{IDH} 1$
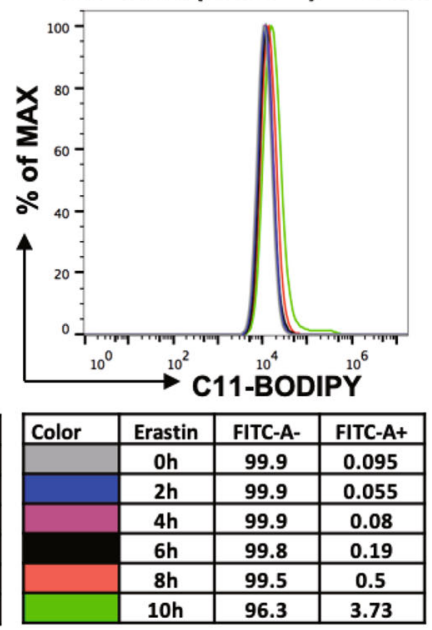

\begin{tabular}{|l|l|l|}
\hline $10 \mathrm{~h}$ & 96.3 & 3.73 \\
\hline
\end{tabular}

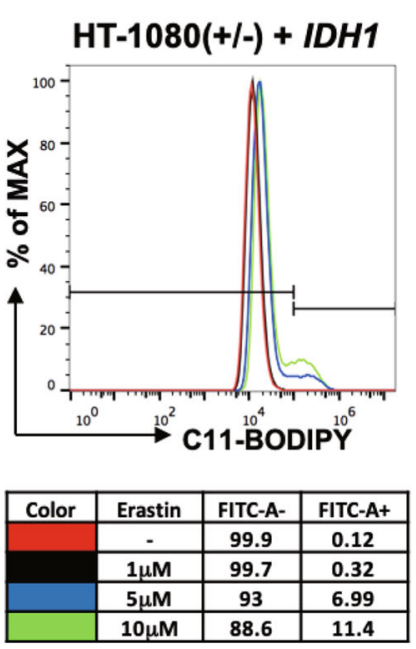

C

$\mathrm{HT}-1080\left(\mathrm{IDH} 1^{++-}\right)+\mathrm{IDH} 1$

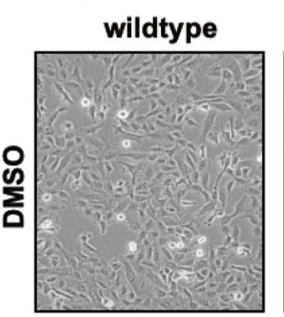
R132C
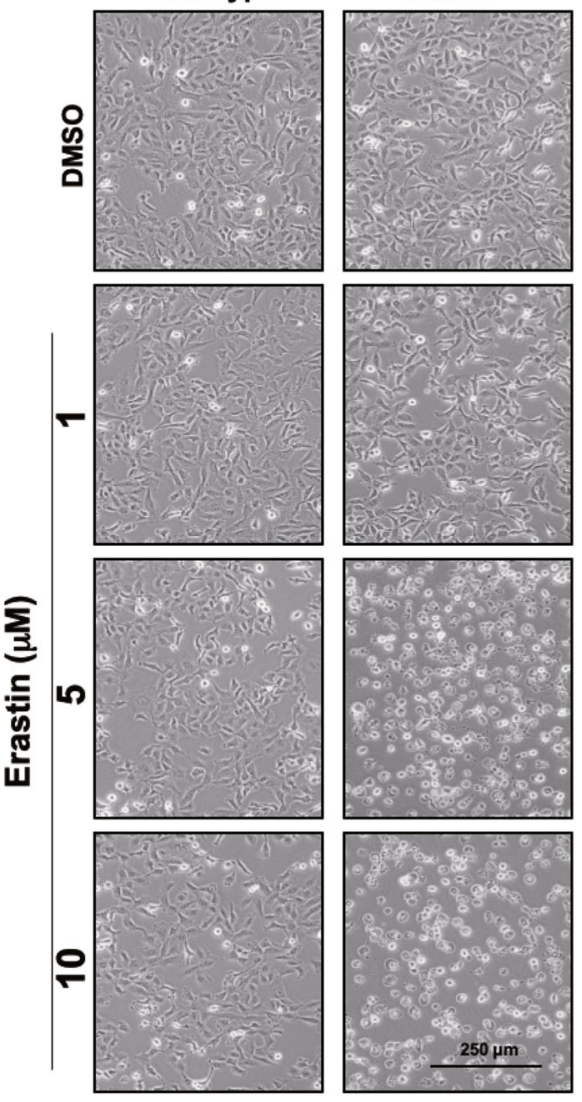

D $H T-1080\left(I D H 1^{+/ R 132 C}\right)$

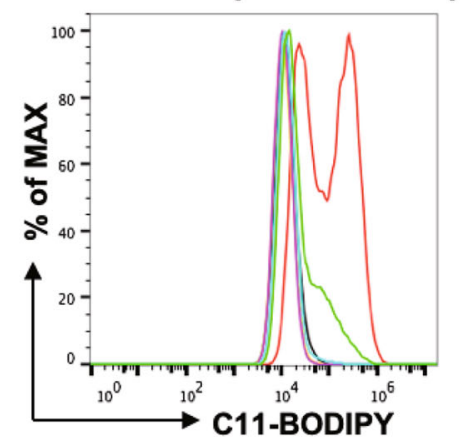

\begin{tabular}{|c|c|c|c|}
\hline & Treatment & FITC-A- & FITC-A- \\
\hline & Ctr & 99.8 & 0.015 \\
\hline & Erastin & 51.3 & 48.7 \\
\hline & AG120 pretreat Ctr & 99.9 & 0.096 \\
\hline & AG120 pretreat Erastin & 89.3 & 10.7 \\
\hline & IDH-889 pretreat Ctr & 99.9 & 0.1 \\
\hline & IDH-889 pretreat Erastin & 99.4 & 0.63 \\
\hline
\end{tabular}


(see figure on previous page)

Fig. 2 Mutant IDH1 enhances erastin-induced lipid ROS accumulation. a IDH1 ${ }^{\mathrm{R} 132 \mathrm{C}}$ mutation enhances erastin-induced lipid ROS accumulation in a time-dependent manner. HT-1080 $\left(\mathrm{IDH} \mathrm{I}^{+/-}\right.$) cells expressing ectopic IDH1 or IDH1 ${ }^{\mathrm{R} 132 \mathrm{C}}$ were treated with erastin $(10 \mu \mathrm{M})$ for indicated time and the accumulation of lipid ROS was assessed by C11 BODIPY 581/591 staining coupled with flow cytometry analysis. b Mutant IDH1 enhances erastininduced lipid ROS accumulation in a concentration-dependent manner. $\mathrm{HT}$-1080(IDH1 ${ }^{+/-}$) cells expressing ectopic IDH1 or IDH1 ${ }^{\mathrm{R} 132 \mathrm{C}}$ were treated with different concentrations of erastin for $10 \mathrm{~h}$ and the accumulation of lipid ROS within cells was assessed by C11 BODIPY 581/591 staining coupled with flow cytometry analysis. c Cell state in (b) was captured by microscope. $\mathbf{d}$ Mutant IDH1 inhibitors inhibit erastin-induced lipid ROS accumulation. HT-1080(IDH $\left.1^{+/ R 132 C}\right)$ cells were treated with mutant IDH1 inhibitors AG-120 or IDH-889 for $12 \mathrm{~h}$, and then with erastin for additional $10 \mathrm{~h}$. The accumulation of lipid ROS within cells was assessed by C11 BODIPY 581/591 staining coupled with flow cytometry analysis

$\left(\mathrm{IDH} 1^{+/-}\right)$cells survived (Fig. 5b). These data indicate that ferroptosis is a reversible process until a critical point is reached.

In searching for the mechanism underlying the effect that mutant IDH1 enhance ferroptosis, we found, surprisingly, that unlike the response to erastin, HT-1080 $\left(I D H 1^{+/ R 132 C}\right)$ and HT-1080 $\left(I D H 1^{+/-}\right)$cells showed no difference to the treatment of RSL3, a direct inhibitor of GPX4 that rapidly induces ferroptosis (Fig. 5c). This result suggests that mutant IDH1 may enhance ferroptosis through targeting GPX4. GPX4 is the only known enzyme in mammalian cells that can eliminate lipid ROS. We found that the protein level of GPX4 is up-regulated when $I D H 1^{\text {R132C }}$ was deleted (Fig. 5d). We confirmed the effect of mutant IDH1 on GPX4 protein level by the observation that overexpression of D2HGDH in HT-1080 $\left(\mathrm{IDH} 1^{+/ \mathrm{R} 132 \mathrm{C}}\right)$, which cleared out D-2-HG produced by mutant IDH1, upregulated GPX4 (Fig. 5e). Conversely, treatment of HT-1080 $\left(I D H 1^{+/-}\right)$cells with cellpermeable D-2-HG downregulated the protein level of GPX4 (Fig. 5f). These observations reveal a possible molecular mechanism that IDH1 $1^{\mathrm{R} 132 \mathrm{C}}$ mutation sensitizes cells to erastin-induced ferroptosis through regulating the protein level of GPX4.

\section{Discussion}

Since ferroptosis was defined in $2012^{3}$, much effort has been devoted to elucidate the mechanisms underlying this new form of regulated cell death. Several major aspects involved in ferroptosis regulation have been identified, including iron metabolism, lipid oxidation, and redox homeostasis $^{32}$. However, a key unanswered question is why different cell lines exhibit markedly different sensitivities to the same ferroptosis signals. This suggests that ferroptosis is highly cell-context dependent and is likely affected by different genetic backgrounds. In this study, we discover that the IDH1 mutation borne by HT-1080 cells, a model cell line commonly used for ferroptosis studies, contributes to the high sensitivity of HT-1080 cells to ferroptosis-inducing agent. Deletion of the mutant $I D H 1$ allele rendered cells less sensitive to erastin-induced ferroptosis, and conversely, re-expression of mutant, but not WT IDH1 re-sensitized HT-1080 $\left(\mathrm{IDH} 1^{+/-}\right)$cells to ferroptosis. We further demonstrated that D-2-HG, the product of mutant IDH1, mediates the effect of mutant IDH1 in sensitizing HT-1080 cells to ferroptosis. In accordance, inhibitors of mutant IDH1 or overexpression of D2HGDH, reduced cells' sensitivity to erastin-induced ferroptosis. These findings uncover a previously unrecognized activity of IDH mutation in promoting ferroptosis. It was recently reported that that inhibition of mitochondrial TCA cycle or electron transfer chain (ETC) reduces lipid peroxide accumulation and ferroptosis ${ }^{33}$. Our study adds another important evidence supporting the notion that ferroptosis may be enhanced by altered metabolism linked to pathological mutation.

Mutant IDH1/2 gains a neomorphic activity of converting $\alpha-K G$ to 2 -HG. Two other metabolites, fumarate, and succinate, are found to accumulate in cells with mutations in succinate dehydrogenase (SDH) or fumarate hydratase $(\mathrm{FH})$. One common mechanism underlying these three metabolites is that these oncometabolites, because of their structural similarity with $\alpha-K G$, promote cell transformation through acting as an antagonist of $\alpha$ $K G$ and competitively inhibiting $\alpha-K G$-dependent dioxygenases, such as histone and DNA demethylases. Interestingly, a recent study reported that $F H$ mutation renders cells more resistant to cysteine-starvation induced ferroptosis $^{34}$. The authors suggest that loss of FH activity accumulates fumarate and disrupts TCA cycle, leading to cell resistance to ferroptosis. In contrast, our study found IDH1 mutation, which accumulates oncometabolite D-2HG, increases cells' sensitivity to erastin-induced ferroptosis. Hence, D-2-HG affects ferroptosis differently from fumarate, suggesting the possibility that these two metabolites may affect ferroptosis via a mechanism independent of inhibiting an $\alpha$-KG-dependent dioxygenase(s). For example, IDH mutation has been reported to cause respiration inhibition through inducing hypersuccinylation in the mitochondria ${ }^{34}$. Since normal TCA cycle is essential for ferroptosis ${ }^{33}$, we speculate that 2-HG sensitizes cell to ferroptosis through a mechanism linked to mitochondrial function. Indeed, we found IDH1 mutation leads to more rapid accumulation of lipid ROS, which is believed to be the key inducer of ferroptosis. Impaired glutathione homeostasis may contribute to this 


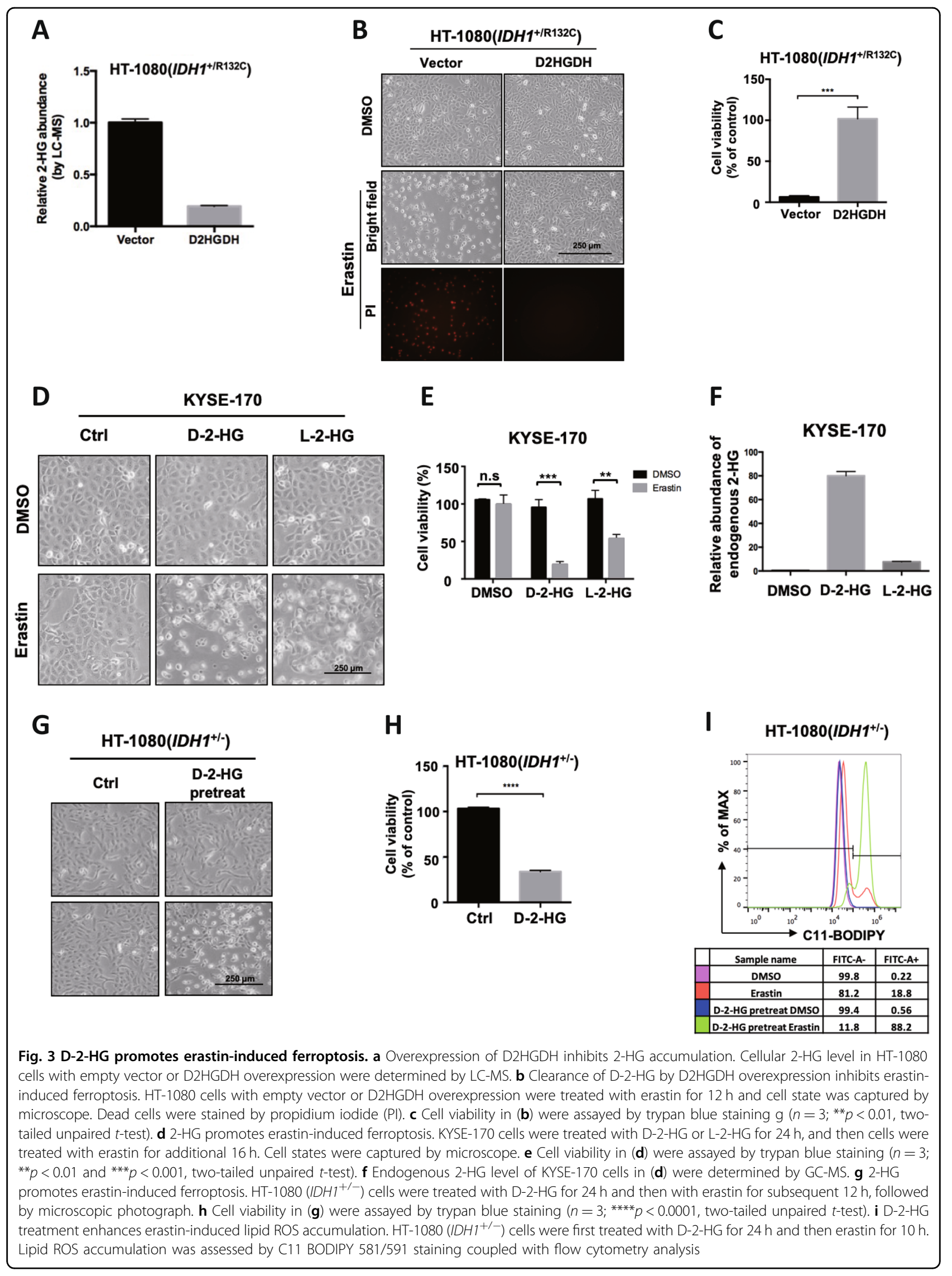



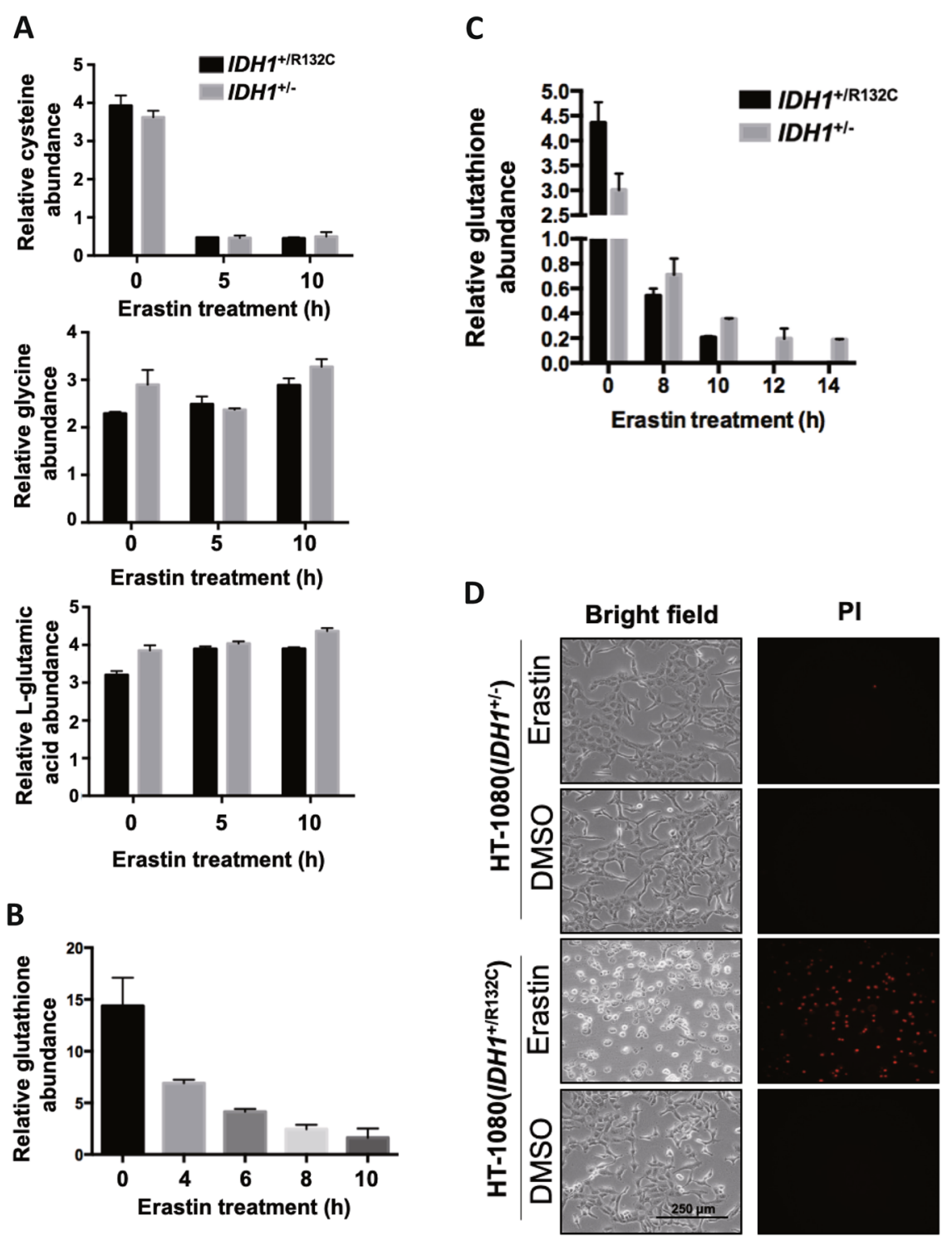

Fig. 4 Mutant IDH1 exacerbates erastin-induced glutathione depletion. a Measurement of cellular levels of glycine, cysteine and L-glutamic

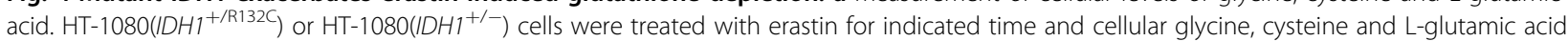
levels were determined by GC-MS. $\mathbf{b}$ Erastin treatment reduces cellular glutathione level over time. $\mathrm{HT}-1080\left(\mathrm{IDH} 1^{+/ \mathrm{R} 132 \mathrm{C}}\right)$ cells were treated with erastin for indicated time and cellular glutathione levels were measured using Total Glutathione Assay Kit. c Mutant IDH1 exacerbates erastin-induced

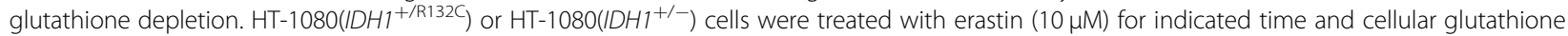
levels were measured using Total Glutathione Assay Kit. $\mathbf{d ~ H T}-1080\left(I D H 1^{+/ R 132 C}\right)$ or $\mathrm{HT}-1080\left(I D H 1^{+-}\right)$cells were treated with erastin for $12 \mathrm{~h}$ and cells were microscopically photographed

phenomenon, evidenced by a faster exhaustion of glutathione in HT-1080(IDH1 $\left.{ }^{+/ R 132 C}\right)$ cells upon erastin treatment

(Fig. 4c).

Notably, IDH1 mutation or 2-HG treatment leads to downregulation of GPX4 protein level and elimination of mutant IDH1 or overexpression of D2HGDH upregulated GPX4 protein level. Interestingly, we further observed that IDH1 mutation does not affect RSL3 induced ferroptosis (Fig. 5c), supporting the notion that IDH1 mutation sensitize cells to ferroptosis through GPX4. RSL3 inhibits GPX4 in a concentration-dependent manner. It is notable that previous studies showed cells with GPX4 overexpression by several folds were less sensitive to $\mathrm{RSL}^{30}$. In our experiment, however, high 2-HG level decreased GPX4 protein level by about fifty percent (Fig. 5d). In this case, the inhibitory effect of RSL3 on GPX4 would be exacerbated. We used RSL3 at a concentration of $2 \mu \mathrm{M}$, which is generally used for inhibiting GPX4 and rapidly inducing ferroptosis in HT-1080 cells, 


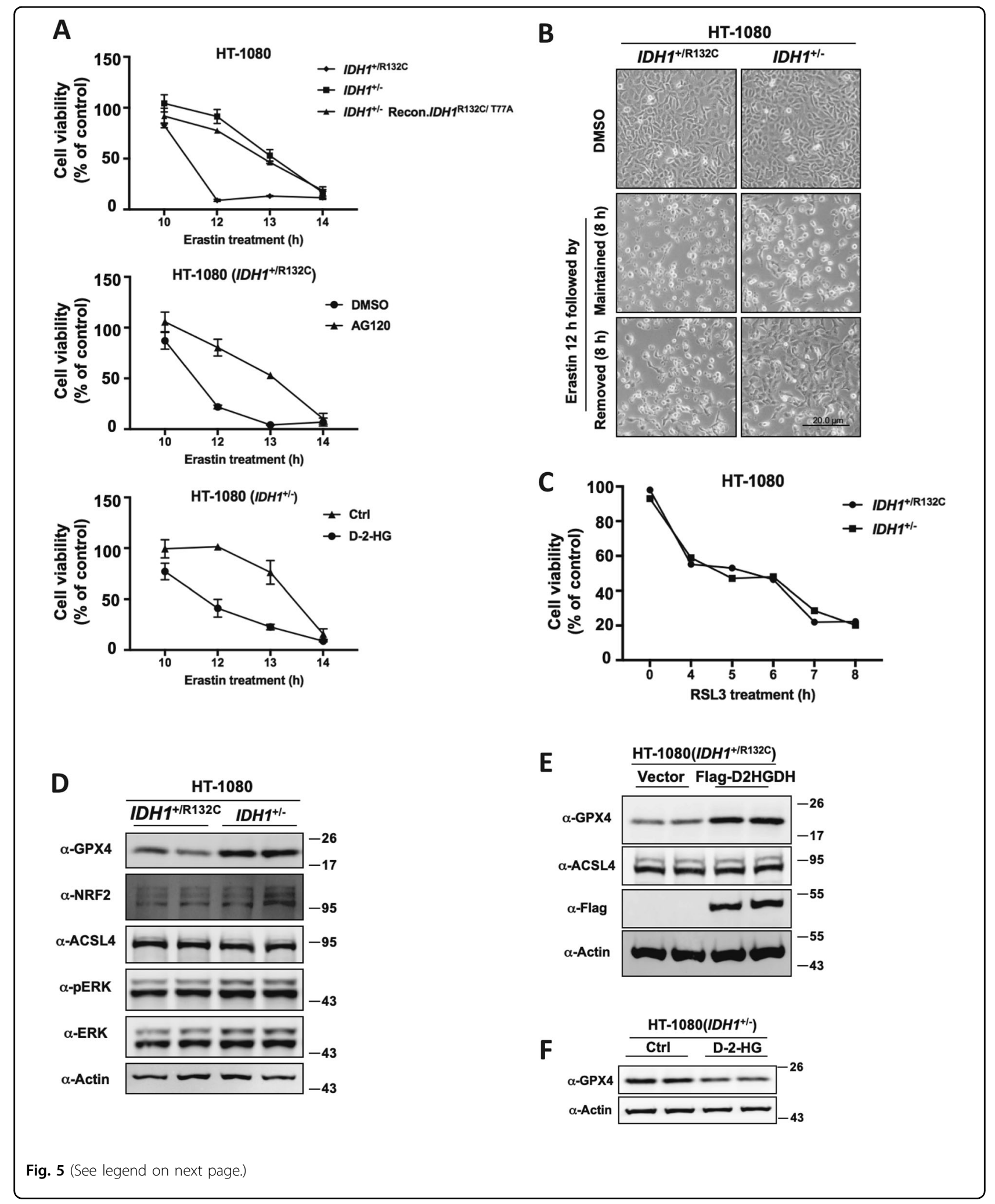

to demonstrate that full suppression of GPX4 made cells insensitive to 2-HG, indicating that IDH1 mutation or 2HG accumulation may affect ferroptosis through regulating GPX4. GPX4 is thus far the only known enzyme in mammalian cells to eliminate lipid ROS and thus its expression level and activity determines cell fate 
(see figure on previous page)

Fig. 5 IDH1 mutation reduces GPX4 protein level. a IDH1 ${ }^{\mathrm{R} 132 \mathrm{C}}$ mutation and 2-HG treatment accelerate the onset of erastin-induced ferroptosis. HT-1080 cells with indicated genotypes or treatment were treated with erastin $(10 \mu \mathrm{M})$ for indicated time, and cell viability were assayed by trypan blue staining. $\mathbf{b}$ Ferroptosis is a reversible process before reaching a point of no-return. $\mathrm{HT}-1080\left(\mathrm{IDH} 1^{+/ \mathrm{R} 132 \mathrm{C}}\right)$ or $\mathrm{HT}-1080\left(\mathrm{IDH} \mathrm{1}^{+/-}\right)$cells were treated with erastin for $12 \mathrm{~h}$ and then erastin was either removed from or kept in the culture medium for additional $8 \mathrm{~h}$, followed by microscopic photograph. c Mutant IDH1 does not enhance ferroptosis in cells treated with GPX4 inhibitor RSL3. HT-1080(IDH1 ${ }^{+/ R 132 \mathrm{C}}$ ) or HT-1080(IDH1 $\left.{ }^{+/-}\right)$cells were treated with RSL3 $(2 \mu \mathrm{M})$ for indicated time, and cell viability were assayed by trypan blue staining. $\mathbf{d}$ Knocking out of IDH1R132C allele

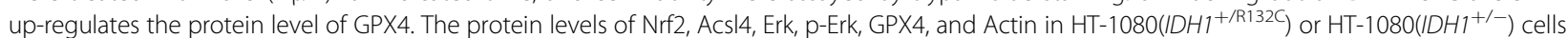
were detected by western blot. e D2HGDH overexpression upregulates the protein level of GPX4. The protein level of Acs/4, Flag-D2HGDH, GPX4 and Actin in $\mathrm{HT}-1080(\mathrm{IDH})^{+/ \mathrm{R} 132 \mathrm{C}}$ ) cells expressing vector or Flag-D2HGDH were detected by western blot. $\mathbf{f} \mathrm{D}-2-\mathrm{HG}$ treatment down-regulates the protein level of GPX4. The protein level of GPX4 and Actin in HT-1080 $\left(I D H 1^{+/-}\right)$cells with or without D-2-HG treatment were detected by western blot

upon ferroptotic signals. It is conceivable that IDH1 mutation and D-2-HG regulate the expression of GPX4.

In addition to monotherapy targeting mutant IDH enzymes through pharmacological inhibition, multiple combination therapies are being explored to target the vulnerability of IDH-mutant tumors. These include increased sensitivity of IDH-mutant tumors to alkylating agents $^{35,36}$, to inhibitors of nicotinamide phosphoribosyltransferase (NAMPT) ${ }^{37}$, and inhibitor of poly ADP ribose polymerase (PARP $)^{38}$. Our findings suggest that sensitizing cells to ferroptosis may be worth to explore for the treatment of IDH-mutant tumors.

\section{Acknowledgements}

This work was supported by National Key Research and Development Project of China (No. 2018YFA0800304 to H.-X.Y.), National Science and Technology Major Project of China (No. 2017ZX10203207-002-004 to H.-X.Y.) and National Natural Science Foundation of China Grant (No. 31970684 and 81773190 to H.-X.Y.).

\section{Author details}

${ }^{1}$ The Fifth People's Hospital of Shanghai and the Molecular and Cell Biology Research Lab of the Institutes of Biomedical Sciences, Fudan University, Shanghai, China. ²Department of Biochemistry and Biophysics, Lineberger Comprehensive Cancer Center, University of North Carolina at Chapel Hill, Chapel Hill, North Carolina, USA. ${ }^{3}$ Department of Pharmacology and Moores Cancer Center, University of California San Diego, La Jolla, California, USA

\section{Conflict of interest}

Kun-Liang Guan is a co-founder of Vivace Therapeutics. Yue Xiong is a cofounder of Cullgen Inc. The remaining authors declare that they have no conflict of interest.

\section{Publisher's note}

Springer Nature remains neutral with regard to jurisdictional claims in published maps and institutional affiliations.

Received: 20 July 2019 Revised: 30 August 2019 Accepted: 12 September 2019

Published online: 07 October 2019

\section{References}

1. Dolma, S., Lessnick, S. L., Hahn, W. C. \& Stockwell, B. R. Identification of genotype-selective antitumor agents using synthetic lethal chemical screening in engineered human tumor cells. Cancer Cell 3, 285-296 (2003).
2. Soulinna, E. M. \& Winberg, A. M. 7-Ethoxycoumarin O-deethylation and methylumbelliferone conjugation in isolated fetal and adult bovine hepatocytes. Drug Metab. Disposition: Biol. Fate Chem. 13, 722-724 (1985).

3. Dixon, S. J. et al. Ferroptosis: an iron-dependent form of nonapoptotic cell death. Cell 149, 1060-1072 (2012).

4. Angeli, J. P. F., Shah, R., Pratt, D. A. \& Conrad, M. Ferroptosis inhibition: mechanisms and opportunities. Trends Pharmacol. Sci. 38, 489-498 (2017).

5. Shah, R., Shchepinov, M. S. \& Pratt, D. A. Resolving the role of lipoxygenases in the initiation and execution of ferroptosis. ACS Cent. Sci. 4, 387-396 (2018).

6. Doll, S. et al. ACSL4 dictates ferroptosis sensitivity by shaping cellular lipid composition. Nat. Chem. Biol. 13, 91-98 (2017).

7. Gao, M., Monian, P., Quadri, N., Ramasamy, R. \& Jiang, X. Glutaminolysis and transferrin regulate ferroptosis. Mol. Cell 59, 298-308 (2015).

8. Lecher, P. \& Signoret, J. Current data on sex chromosomes in mammals. L' Annee Biologique 8, 167-198 (1969).

9. Lewerenz, J., Ates, G., Methner, A., Conrad, M. \& Maher, P. Oxytosis/ ferroptosis-(Re-) emerging roles for oxidative stress-dependent nonapoptotic cell death in diseases of the central nervous system. Front. Neurosci. 12, 214 (2018).

10. Friedmann Angeli, J. P. et al. Inactivation of the ferroptosis regulator Gpx4 triggers acute renal failure in mice. Nat. Cell Biol. 16, 1180-1191 (2014).

11. Linkermann, A. et al. Synchronized renal tubular cell death involves ferroptosis. Proc. Natl Acad. Sci. USA 111, 16836-16841 (2014).

12. Parsons, D. W. et al. An integrated genomic analysis of human glioblastoma multiforme. Science 321, 1807-1812 (2008).

13. Mardis, E. R. et al. Recurring mutations found by sequencing an acute myeloid leukemia genome. New Engl. J. Med. 361, 1058-1066 (2009).

14. Amary, M. F. et al. IDH1 and IDH2 mutations are frequent events in central chondrosarcoma and central and periosteal chondromas but not in other mesenchymal tumours. J. Pathol. 224, 334-343 (2011).

15. Pansuriya, T. C. et al. Somatic mosaic IDH1 and IDH2 mutations are associated with enchondroma and spindle cell hemangioma in Ollier disease and Maffucci syndrome. Nat. Genet. 43, 1256-1261 (2011).

16. Amary, M. F. et al. Ollier disease and Maffucci syndrome are caused by somatic mosaic mutations of IDH1 and IDH2. Nat. Genet. 43, 1262-1265 (2011).

17. Wang, P. et al. Mutations in isocitrate dehydrogenase 1 and 2 occur frequently in intrahepatic cholangiocarcinomas and share hypermethylation targets with glioblastomas. Oncogene 32, 3091-3100 (2013).

18. Borger, D. R. et al. Frequent mutation of isocitrate dehydrogenase (IDH) 1 and $\mathrm{IDH} 2$ in cholangiocarcinoma identified through broad-based tumor genotyping. Oncologist 17, 72-79 (2012).

19. Ye, D., Guan, K. L. \& Xiong, Y. Metabolism, activity, and targeting of D- and L-2hydroxyglutarates. Trends Cancer 4, 151-165 (2018).

20. Dang, L. et al. Cancer-associated IDH1 mutations produce 2-hydroxyglutarate. Nature 465, 966 (2010).

21. $\mathrm{Xu}, \mathrm{W}$. et al. Oncometabolite 2-hydroxyglutarate is a competitive inhibitor of alpha-ketoglutarate-dependent dioxygenases. Cancer Cell 19, 17-30 (2011).

22. Chowdhury, R. et al. The oncometabolite 2-hydroxyglutarate inhibits histone lysine demethylases. EMBO Rep. 12, 463-469 (2011).

23. Fu, X. et al. 2-Hydroxyglutarate inhibits ATP synthase and mTOR signaling. Cell Metab. 22, 508-515 (2015).

24. Chin, R. M. et al. The metabolite alpha-ketoglutarate extends lifespan by inhibiting ATP synthase and TOR. Nature 510, 397-401 (2014). 
25. Stockwell, B. R. et al. Ferroptosis: a regulated cell death nexus linking metabolism, redox biology, and disease. Cell 171, 273-285 (2017).

26. Jin, $\mathrm{G}$. et al. Mutant IDH1 is required for IDH1 mutated tumor cell growth. Oncotarget 3, 774-782 (2012).

27. $\mathrm{Ma}$, S. et al. D-2-hydroxyglutarate is essential for maintaining oncogenic property of mutant IDH-containing cancer cells but dispensable for cell growth. Oncotarget 6, 8606-8620 (2015).

28. Popovici-Muller, J. et al. Discovery of AG-120 (Ivosidenib): a first-in-class mutant $\mathrm{IDH} 1$ inhibitor for the treatment of $\mathrm{IDH} 1$ mutant cancers. ACS Med. Chem. Lett 9, 300-305 (2018).

29. Xie, $X$. et al. Allosteric mutant IDH1 inhibitors reveal mechanisms for IDH1 mutant and isoform selectivity. Structure 25, 506-513 (2017).

30. Yang, W. S. et al. Regulation of ferroptotic cancer cell death by GPX4. Cell 156 317-331 (2014).

31. Hayes, J. D. \& McLellan, L. I. Glutathione and glutathione-dependent enzymes represent a co-ordinately regulated defence against oxidative stress. Free Radic. Res. 31, 273-300 (1999).
32. Conrad, $M$. et al. Regulation of lipid peroxidation and ferroptosis in diverse species. Genes Dev. 32, 602-619 (2018).

33. Gao, M. et al. Role of mitochondria in ferroptosis. Mol. Cell 73, 354-363 e353 (2019).

34. Li, F. et al. NADP(+)-IDH mutations promote hypersuccinylation that impairs mitochondria respiration and induces apoptosis resistance. Mol. Cell $\mathbf{6 0}$ 661-675 (2015).

35. Cairncross, J. G. et al. Benefit from procarbazine, lomustine, and vincristine in oligodendroglial tumors is associated with mutation of IDH. J. Clin. Oncol. 32, 783-790 (2014).

36. Buckner, J. C. et al. Radiation plus Procarbazine, CCNU, and Vincristine in LowGrade Glioma. N. Engl. J. Med. 374, 1344-1355 (2016).

37. Tateishi, $\mathrm{K}$. et al. Extreme Vulnerability of IDH1 Mutant Cancers to NAD+ Depletion. Cancer Cell 28, 773-784 (2015).

38. Sulkowski, P. L. et al. 2-Hydroxyglutarate produced by neomorphic IDH mutations suppresses homologous recombination and induces PARP inhibitor sensitivity. Sci. Transl. Med. 9, pii: eaal2463 (2017). 